\title{
On the fraction of X-ray obscured quasars in the local universe
}

\author{
J. X. Wang \& P. Jiang \\ Center for Astrophysics, University of Science and Technology of China, Hefei, Anhui \\ 230026, P. R. China \\ Joint Institute of Galaxies and Cosmology, USTC and SHAO, CAS \\ jxw@ustc.edu.cn
}

\begin{abstract}
Recent wide area hard X-ray and soft Gamma ray surveys have shown that the fraction of X-ray obscured AGNs in the local universe significantly decreases with intrinsic luminosity. In this letter we point out that two correction have to be made to the samples: 1) radio loud AGNs have to be excluded since their X-ray emission might be dominated by the jet component; 2) Compton thick sources have to be excluded too since their hard X-ray and soft gamma ray emission are also strongly attenuated by Compton scattering. The soft gammaray selected AGN samples obtained by SWIFT and INTEGRAL provide the best opportunity to study the fraction of obscured AGN in the local universe in the least biased way. We choose these samples to check if the corrections could alter the above result on the fraction of obscured AGNs. We find that before the corrections both samples show significant anti-correlation between $L_{X}$ and $N_{H}$, indicating obvious decrease in the fraction of obscured AGNs with luminosity. However, after the corrections, we find only marginal evidence of anticorrelation (at $98 \%$ confidence level) in the SWIFT sample, and no evidence at all in the INTEGRAL sample which consists of comparable number of objects. We conclude that current samples only show a marginal decrease in the fraction of obscured AGNs in the local universe, and much larger samples are required to reach a more robust conclusion.
\end{abstract}

Subject headings: galaxies: active — quasars: general — X-rays: galaxies

\section{Introduction}

A large population of obscured powerful quasars called type- 2 quasars has long been predicted by the widely accepted unified model for active galactic nuclei (AGNs; Antonucci 
1993). They are believed to be intrinsically the same as type-1 quasars but with strong obscuration in both optical and X-ray band due to the presumable torus. Most of such type-2 quasars, which might dominate the black hole growth (e.g., Martínez-Sansigre et al. 2005), have been missed by optical surveys for quasars. Doubt has been expressed at times on the existence of type-2 quasars (e.g. Halpern, Turner \& George 1999), mainly due to their rareness and the fact that they are easily mimicked by other types of AGNs. The hard $\mathrm{X}$-ray emission is less affected by the photon-electric absorption, making hard X-ray surveys good approach to search for type-2 quasars. Another advantage of X-day data is that Xray spectral fitting can yield intrinsic luminosity and absorption simultaneously, which are both required to study the population of obscured quasars. A number of obscured quasars have been successfully revealed by recent deep and wide-area X-ray surveys performed by Chandra and XMM (Norman et al. 2002; Stern et al. 2002; Mainieri et al. 2002; Fiore et al. 2003; Caccianiga et al. 2004).

However, many studies have claimed that the fraction of obscured quasars is much smaller than expected from the simplified AGN unified model. Ueda et al. (2003) computed the X-ray luminosity function for $2-10 \mathrm{keV}$ selected AGN samples, and found that the fraction of X-ray absorbed AGNs (with $N_{H}>10^{22} \mathrm{~cm}^{-2}$ ) drops from $\sim 0.6$ at intrinsic $L_{X}$ around $10^{42} \mathrm{ergs} \mathrm{s}^{-1}$ to around 0.3 at $L_{X}$ above $10^{44} \mathrm{ergs} \mathrm{s}^{-1}$. Several other studies based on hard X-ray surveys also support the scheme that the fraction of type II AGNs (or X-ray absorbed AGNs) decreases with intrinsic luminosity (Steffen et al. 2003; Barger et al. 2005; La Franca et al. 2005; Shinozaki et al. 2006). However, contrary results are also reported. Perola et al. (2004) found that the fraction of obscured AGNs in HELLAS2XMM does not change with X-ray luminosity, although the fraction (40\%) appears smaller than expected. Eckart et al. (2006) found that half of the AGNs identified by the SEXSI program are X-ray obscured with $N_{H}>10^{22} \mathrm{~cm}^{-2}$, and the fraction of obscured AGNs is independent of the unobscured luminosity. The discrepancy can be attributed to the redshift evolution in the fraction of obscured quasars (La Franca et al. 2005; Wang et al. 2006): while shallow and wide surveys are probing quasars in the local universe where probably only a small fraction of quasars are obscured, deep surveys are detecting quasars at much higher redshift where more than half of them are obscured (Wang et al. 2006).

Soft gamma-ray selected AGN samples (Markwardt et al. 2005, hereafter M06; Bassani et al. 2006, hereafter B06; Beckmann, Gehrels \& Shrader 2006) obtained by SWIFT and INTEGRAL provide the best opportunity to study the fraction of obscured AGN in the local universe in the least biased way. Note that for Compton-thick cases $\left(\mathrm{N}_{H}>10^{24}\right.$ $\mathrm{cm}^{-2}$ ), even soft gamma-ray emission could be significantly attenuated, thus soft gamma-ray selected samples are not complete to Compton-thick sources. Claims have been made based on the soft gamma-ray selected samples, that the fraction of obscured AGNs in the local 
universe significantly decrease with increasing luminosity. In this letter we point out that two correction have to be made to the samples to study of the fraction of obscured quasars. The corrections are: a) radio loud AGNs have to be excluded since their X-ray emission might be dominated by the jet component thus the measured luminosity and the obscuration does not reflect the intrinsic values in the nuclei; b) Compton thick sources have to be excluded too since their soft gamma ray emission are also strongly attenuated by Compton scattering and their intrinsic luminosities are hard to estimate. After the corrections, we find only marginal decrease in the fraction of obscured AGN with luminosity in the local universe. Larger samples are required to reach a more robust conclusion.

\section{The Samples}

\subsection{The INTEGRAL Sample}

Bassani et al. (2006) provides an AGN sample selected in the $20-100 \mathrm{keV}$ band with the IBIS on INTEGRAL. The sample contains 62 active galactic nuclei (14 of them are unclassified) above a flux limit of $\sim 1.5 \times 10^{-11} \mathrm{ergs} \mathrm{cm}^{-2} \mathrm{~s}^{-1}$. B06 listed the available column densities (obtained from archival X-ray spectra) for 35 sources with redshifts. In this letter we provide an updated list of $N_{H}$ (and references) with more recent measurements available in literature (mostly from Chandra or XMM observations, Table 1) ${ }^{1}$. Within this subsample, 10 are radio loud (including five blazars), five are Compton-thick $\left(\mathrm{N}_{H} \geqslant 10^{24} \mathrm{~cm}^{-2}\right)$, the rest 20 are radio quiet and Compton-thin. The luminosities in the $20-100 \mathrm{keV}$ band were calculated by assuming a Crab-like spectrum.

\subsection{The SWIFT Sample}

The first 3 months of the SWIFT Burst Alert Telescope (BAT) high Galactic latitude survey provide a sample of $14-195 \mathrm{keV}$ band selected sources (M05) with a flux limit of $\sim 10^{-11} \mathrm{ergs}^{-2} \mathrm{~s}^{-1}$ and $\sim 2^{\prime} .7$ (90\% confidence) positional uncertainties for the faintest sources. $86 \%$ of the 66 high-latitude sources were identified. Twelve are Galactic-type sources, and 44 are identified with known AGNs. The luminosities in the $14-195 \mathrm{keV}$ band were calculated by assuming a typical power-law spectrum $(\Gamma \sim 1.7)$. M05 listed the absorption column density derived from literature or archive X-ray spectra for 39 AGNs. We also provide an updated list of $N_{H}$ (and references) with more recent measurements available

\footnotetext{
${ }^{1}$ We note that simply adopting the $N_{H}$ in B06 does not significantly affect out main results in this paper.
} 
in literature (Table 1). The major changes are that two more Compton-thick sources (NGC 1365 and NGC $7582^{2}$ ) are classified. Within the 39 sources, there are 7 radio loud sources (including three blazars), 4 Compton-thick sources and 28 radio quiet Compton-thin Seyfert galaxies.

\subsection{The Combined Sample}

In order to increase the number of soft Gamma ray selected AGNs, we combine the INTEGRAL Sample and the SWIFT Sample. Note that there are 10 objects which were detected by both of them (see Table 1 ). Using these 10 objects, we obtained a linear scaling factor from the SWIFT luminosity to the INTEGRAL one (see Fig. 1). With the scaling factor, we translate all the SWIFT luminosity in $14-195 \mathrm{keV}$ to the the INTEGRAL luminosity in $20-100 \mathrm{keV}$. The combined sample contains 42 radio quiet Compton-thin objects, 14 radio loud sources (including 6 blazars) and eight Compton-thick objects.

\section{Results and Discussion}

In Fig. 2 we plot the X-ray absorption column density versus the SWIFT luminosity for the 39 AGNs in the SWIFT sample. Consistent with M05, we find a clear drop of $N_{H}$ with increasing luminosity. Since soft gamma-ray selected samples are not biased to obscuration (except for Compton thick sources), the sample selection is identical for both obscured and unobscured sources. We can simply study the correlation between $N_{H}$ and $\mathrm{L}_{X}$ (or the luminosity distribution for obscured/unobscured sources) in the samples without calculating the luminosity function or folding luminosity function model with the sample selection. We performed the Spearman Rank (SR) statistic to give the correlation between $N_{H}$ and $\mathrm{L}_{X}$. We found a significant anti-correlation between $N_{H}$ and $\mathrm{L}_{X}$ at $99.99 \%$ level. We note that while a flux-limited sample would introduce spurious correlation between luminosity and redshift (or between luminosities in different bands), such an effect will not affect our study since the measurement of $N_{H}$ is independent of redshift for the local samples. With the radio loud and Compton thick sources excluded, the confidence level of the anti-correlation significantly drops to $98 \%$. 18 out of 28 radio quiet Compton-thin objects show $N_{H} \gtrsim 10^{22}$ $\mathrm{cm}^{-2}$, yielding a fraction of $64 \%$.

\footnotetext{
${ }^{2}$ Both NGC 1365 and NGC 7582 show quick Compton-thick/Compton-thin transitions, see Risaliti et al. 2005 and Turner et al. 2000.
} 
In Fig. 3 we plot $N_{H}$ versus INTEGRAL luminosity for the 35 AGNs in the INTEGRAL sample. As B06 has claimed, the fraction of absorbed AGNs in the whole sample significantly decreases with the $20-100 \mathrm{keV}$ luminosity. We found an obvious anti-correlation between $N_{H}$ and $\mathrm{L}_{X}$ with a confidence level of $99.7 \%$. However, the anti-correlation is obviously dominated by the radio loud and Compton-thick objects. Excluding radio loud and Compton-thick objects, we found no more evidence of anti-correlation (the null hypothesis gives a probability of 0.92 ). 14 out of 20 radio quiet Compton-thin objects show $N_{H} \gtrsim 10^{22}$ $\mathrm{cm}^{-2}$, yielding a fraction of $70 \%$.

We perform the same statistical analysis to the combined sample described in $\S 2$ (see Fig. 4). Similarly, we find strong anti-correlation (with confidence level $>99.99 \%$ ) between $N_{H}$ and $\mathrm{L}_{X}$ in the whole sample, but the anti-correlation is mainly due to the radio loud and Compton-thick objects. With them excluded, the confidence level of the anti-correlation drops to $94 \%$. A KS test also gives similar results that the luminosity distribution of obscured sources (with $N_{H} \gtrsim 10^{22} \mathrm{~cm}^{-2}$ ) differs from that of unobscured ones at confidence level of $>99.99 \%$ and $95 \%$ respectively before and after excluding the radio loud and Compton thick sources. In the combined sample, 29 out of 42 radio quite Compton-thin objects show $N_{H}$ $\gtrsim 10^{22} \mathrm{~cm}^{-2}$, yielding a fraction of $66.7 \%$. For 8 luminous objects with $\mathrm{L}_{20-100 \mathrm{keV}}>10^{44}$ erg s$~^{-1}, 3$ show $N_{H}$ above $10^{22} \mathrm{~cm}^{-2}$, yielding a fraction of $38 \%$. The drop of the fraction is marginal with a confidence level of $91 \%$, consistent with those from the correlation test and $\mathrm{KS}$ test. Note that assuming a powerlaw spectrum with photon index of $\sim 2.0, \mathrm{~L}_{20-100 \mathrm{keV}}$ is directly comparable to $\mathrm{L}_{2-10 k e V}$.

A recent study by Shinozaki et al. (2006) built a complete flux-limited sample of bright AGNs from HEAO-1 all-sky catalogs, with a maximum redshift in the sample of 0.329. Such a sample is ideal to study the population of obscured quasars in the local universe. Shinozaki et al. found no AGN in the high luminosity high-intrinsic absorption regime $\left(\log \mathrm{L}_{X}[\mathrm{erg} / \mathrm{s}]\right.$ $\left.>44.5, \log N_{H}\left[\mathrm{~cm}^{-2}\right]>21.5\right)$ in their sample, where $\sim 5$ AGNs are expected if assuming a constant fraction of obscured AGNs with luminosity. Considering that there are 8 AGNs with $\log \mathrm{L}_{X}[\mathrm{erg} / \mathrm{s}]>44.5$ and $\log N_{H}\left[\mathrm{~cm}^{-2}\right]<21.5$ in their sample, the difference is at a confidence level of $98 \%$. We note that 3 out of 8 sources are obviously radio-loud (3C 273, III Zw2 and PG 1425+267). Removing the radio-loud sources, the confidence level of the difference drops to $90 \%$. This indicates that although the sample presents a trend of decreasing fraction of obscured AGNs with luminosity, the decrease is not statistically solid.

We conclude that the significant decrease with luminosity in the fraction of obscured AGNs in the local universe might be mainly due to the radio loud and Compton-thick objects in the samples. With these objects excluded, we find only marginal evidence of decrease in the SWIFT and Shinozaki sample, but no evidence of decrease in the INTEGRAL sample 
which has a similar sample size. Much larger samples are required to reach a more robust result on the fraction of obscured quasars in the local universe.

The work was supported by Chinese NSF through NSFC10473009, NSFC10533050 and the CAS "Bai Ren" project at University of Science and Technology of China. 


\section{REFERENCES}

Akylas, A. et al. 2002, MNRAS, 332, 23

Antonucci, R. 1993, ARA\&A, 31, 473

Ballantyne, D. R., Fabian, A. C., Iwasawa, K. 2004, MNRAS, 354, 839

Barger, A. J., et al. 2005, ApJ, 129, 578

Bassani, L. et al. 2006, ApJ, 636, L65, B06

Beckmann, V. et al. 2004, ApJ, 614, 641

Beckmann, V. et al. 2005, ApJ, 634, 939

Beckmann, V., Gehrels, N., \& Shrader, C. R. 2006, ApJ, 638, 642

Bianchi, S., Balestra, I., Matt, G., Guainazzi, M., Perola, G. C. 2003, A\&A, 402, 141

Bianchi, S., Miniutti, G., Fabian, A. C., Iwasawa, K. 2005, MNRAS, 360, 380

Caccianiga, A., et a. 2004, A\&A, 416, 901

Colbert, E. J. M., Strickland, D. K., Veilleux, S., Weaver, K. A. 2005, ApJ, 628, 113

Costantini, E., Kaastra, J. S., Steenbrugge, K. C., Arav, N., Gabel, J. R., Kriss, G. 2005, AIPC, 774, 321

Dewangan, G. C., Griffiths, R. E., Schurch, N. J. 2003, ApJ, 592, 52

Eckart, M. E. et al. 2006, ApJ in press, astro-ph0603556

Fiore, F. et al. 2003, A\&A, 409, 79

Foschini, L. et al. 2006, A\&A in press, astro-ph/0603268

Gibson, R. R., Marshall, H. L., Canizares, C. R., Lee, J. C. 2005, ApJ, 627, 83

Halpern, J. P., Turner, T. J., George, I. M. 1999, MNRAS, 307, L47

Jiang, P., Wang, J. X., Wang, T. G. 2006, ApJ in press, astro-ph/0603339

La Franca, F. et al. 2005, ApJ, 635, 864

Lewis, K. T., Eracleous, M., Gliozzi, M., Sambruna, R. M., Mushotzky, R. F. 2005, ApJ, 622,816

Mainieri, V. et al. 2002, A\&A, 393, 425

Markwardt, C. B., Tueller, J., Skinner, G. K., Gehrels, N., Barthelmy, S. D., Mushotzky, R. E. 2005, ApJ, 633, L77, M05

Martínez-Sansigre, A. et al. 2005, Nature, 436, 666

Matsumoto, C. et al. 2004, ApJ, 617, 930 
Matt, G., Bianchi, S., D’Ammando, F., Martocchia, A. 2004, A\&A, 421, 473

Matt, G., Bianchi, S., de Rosa, A., Grandi, P., Perola, G. C. 2006, A\&A, 445, 451

Norman, C. A. et al. 2002, ApJ, 571, 218

Perola, G. C. et al. 2004, A\&A, 421, 491

Risaliti, G., Elvis, M., Fabbiano, G., Baldi, A., Zezas, A. 2005, ApJ, 623, L93

Shinozaki, K., Miyaji, T., Ishisaki, Y., Ueda, Y., Ogasaka, Y. 2006, ApJ in press, astro$\mathrm{ph} / 0603699$

Steenbrugge, K. C. et al. 2003, A\&A, 408, 921

Steenbrugge, K. C. et al. 2005, A\&A, 432, 453

Steffen, A. T., Barger, A. J., Cowie, L. L., Mushotzky, R. F., Yang, Y. 2003, ApJ, 596, L23

Stern, D. et al. 2002, ApJ, 568, 71

Turner, T. J., Perola, G. C., Fiore, F., Matt, G., George, I. M., Piro, L., Bassani, L. 2000, ApJ, 532, 245

Ueda, Y., Akiyama, M., Ohta, K., Miyaji, T. 2003, ApJ, 598, 886

Vaughan, S., Fabian, A. C., Ballantyne, D. R., De Rosa, A., Piro, L., Matt, G. 2004, MNRAS, 351,193

Wang, J. X., et al. 2006, ApJ submitted

Yaqoob, T., Reeves, J. N., Markowitz, A., Serlemitsos, P. J., Padmanabhan, U. 2005, ApJ, 627,156

This preprint was prepared with the AAS IATEX macros v5.2. 


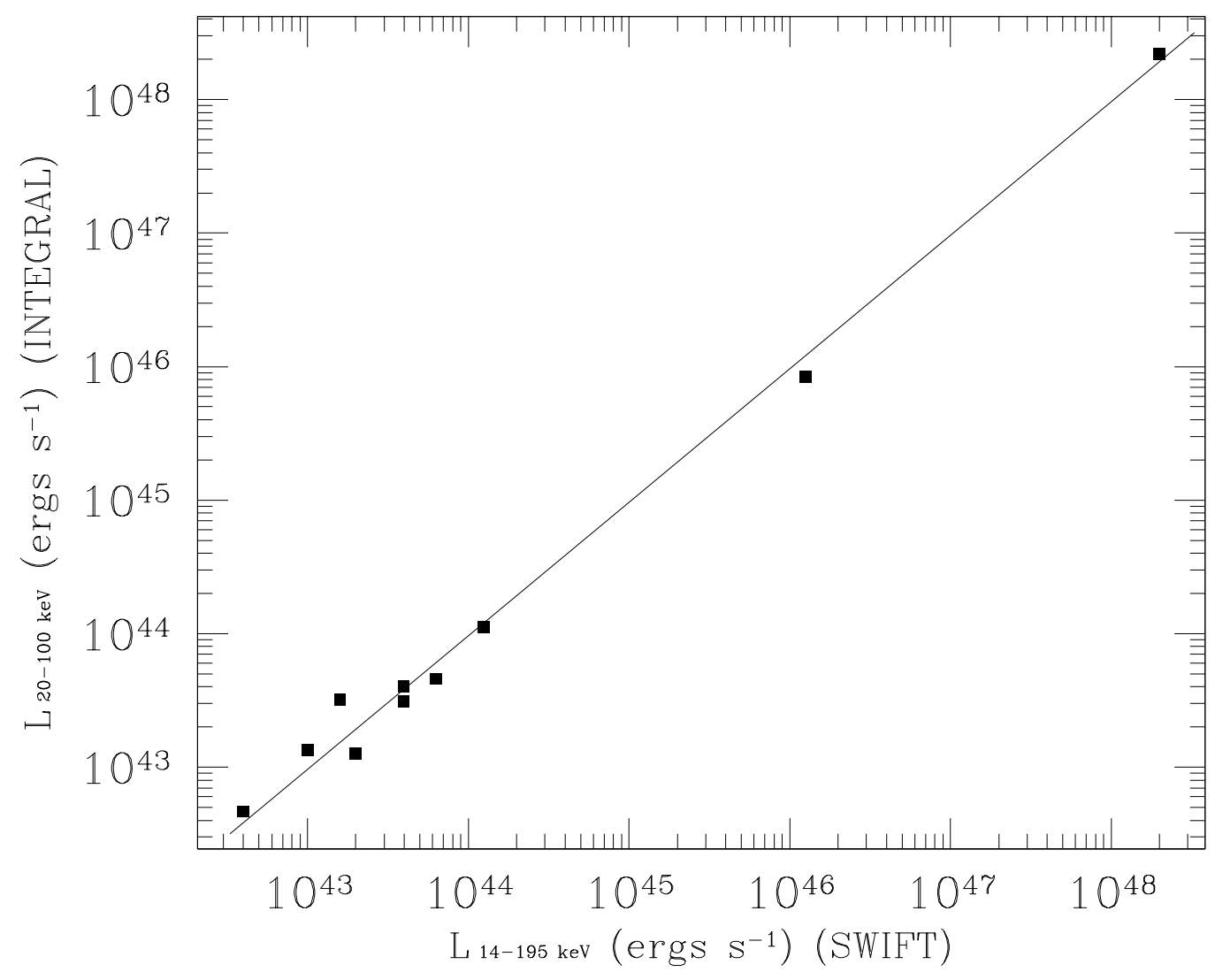

Fig. 1. - The INTEGRAL luminosity in the $20-100 \mathrm{keV}$ band (from Bassani et al. 2006) versus the SWIFT luminosity in the $14-195 \mathrm{keV}$ band (from Markwardt et al. 2005) for 10 AGNs which were detected by both instruments. The solid line shows a linear correlation between two luminosities: $\log \left(\mathrm{L}_{20-100 k e V}\right)=\log \left(\mathrm{L}_{14--195 k \mathrm{kV}}\right)-0.018$. 


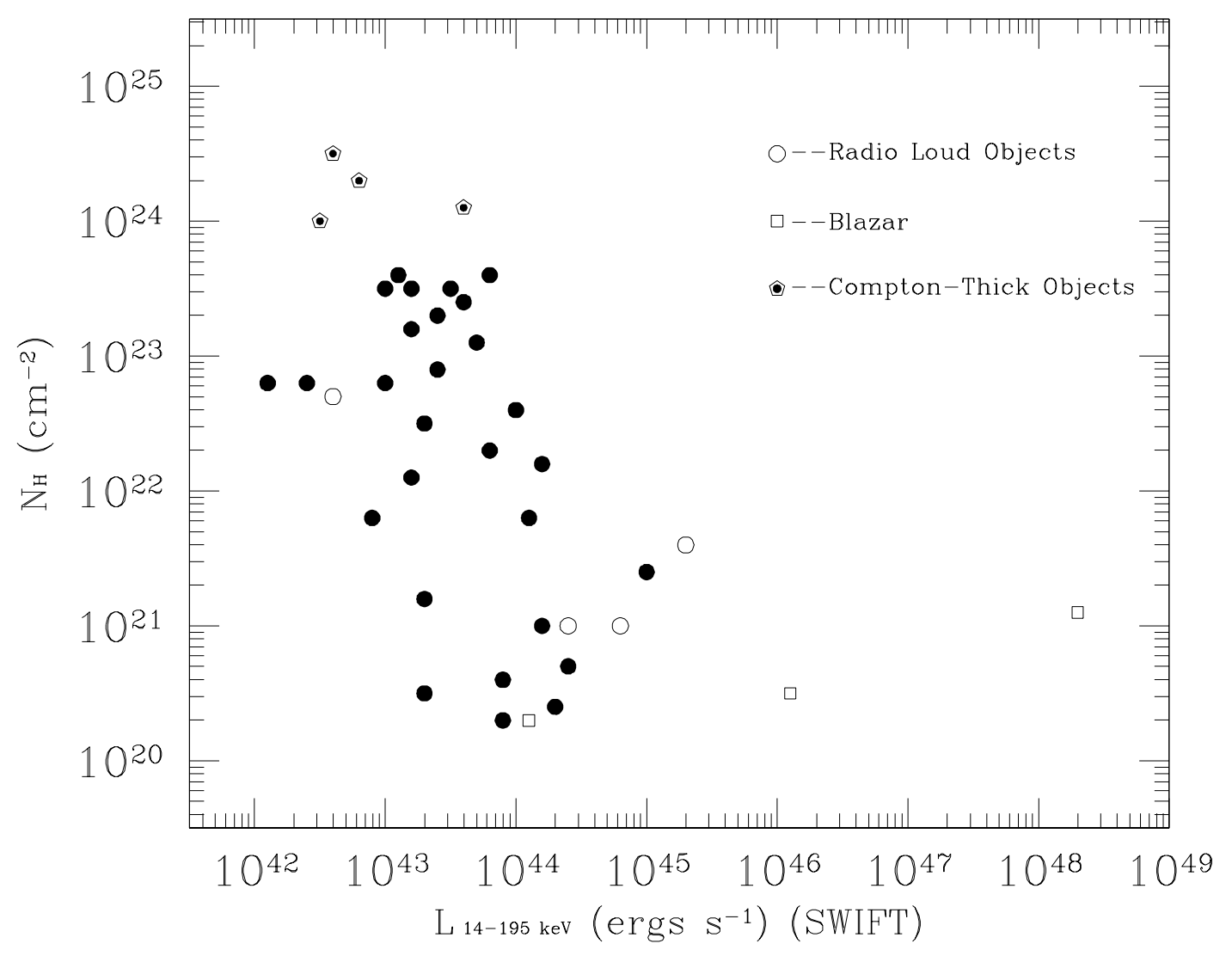

Fig. 2.- The X-ray absorption column density $N_{H}$ versus the SWIFT luminosity in the 14 - $195 \mathrm{keV}$ band for the 39 AGNs in Markwardt et al. (2005). 


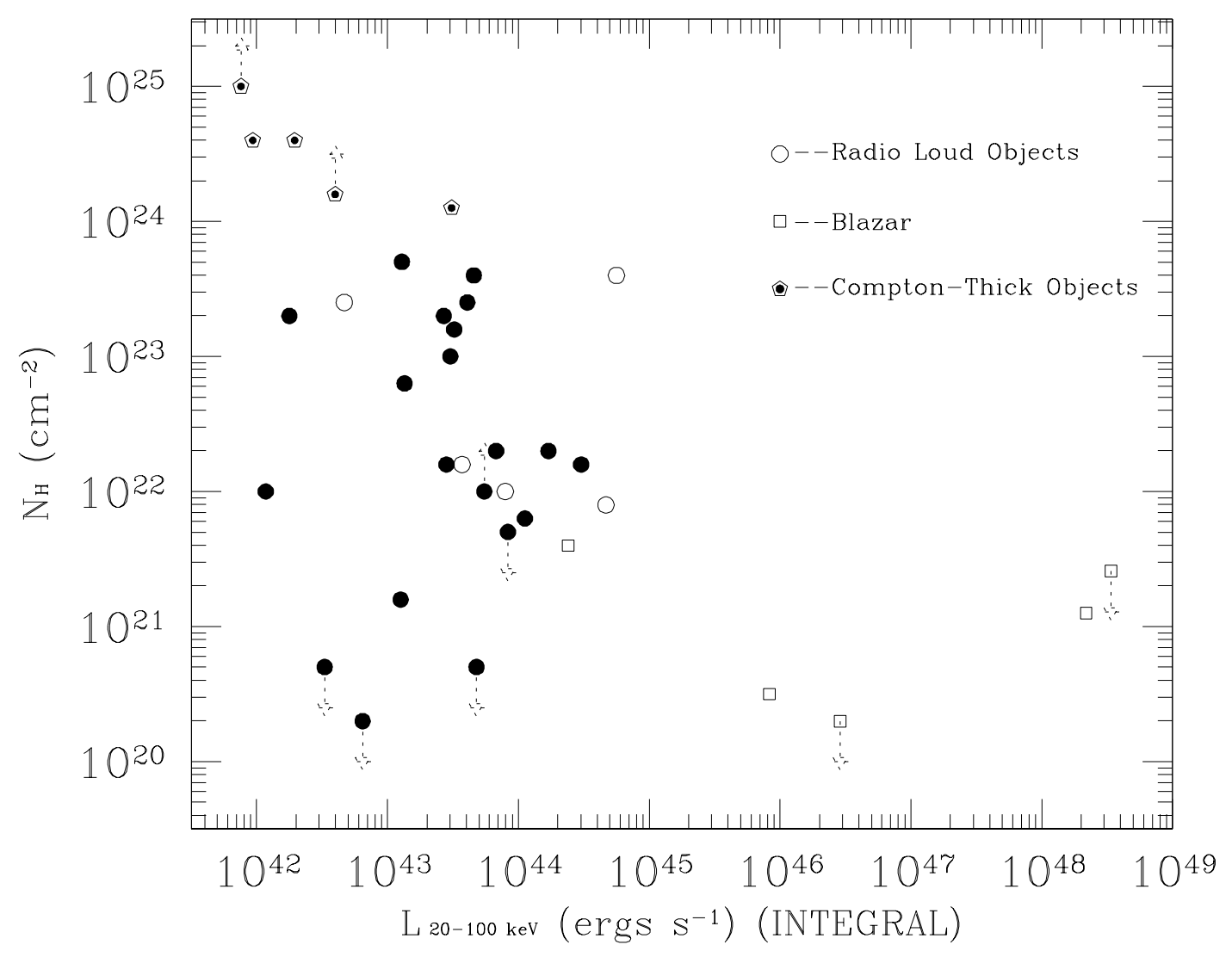

Fig. 3.- The X-ray absorption column density $N_{H}$ versus the INTEGRAL luminosity in the $20-100 \mathrm{keV}$ band for the 35 AGNs in Bassani et al. (2006). 


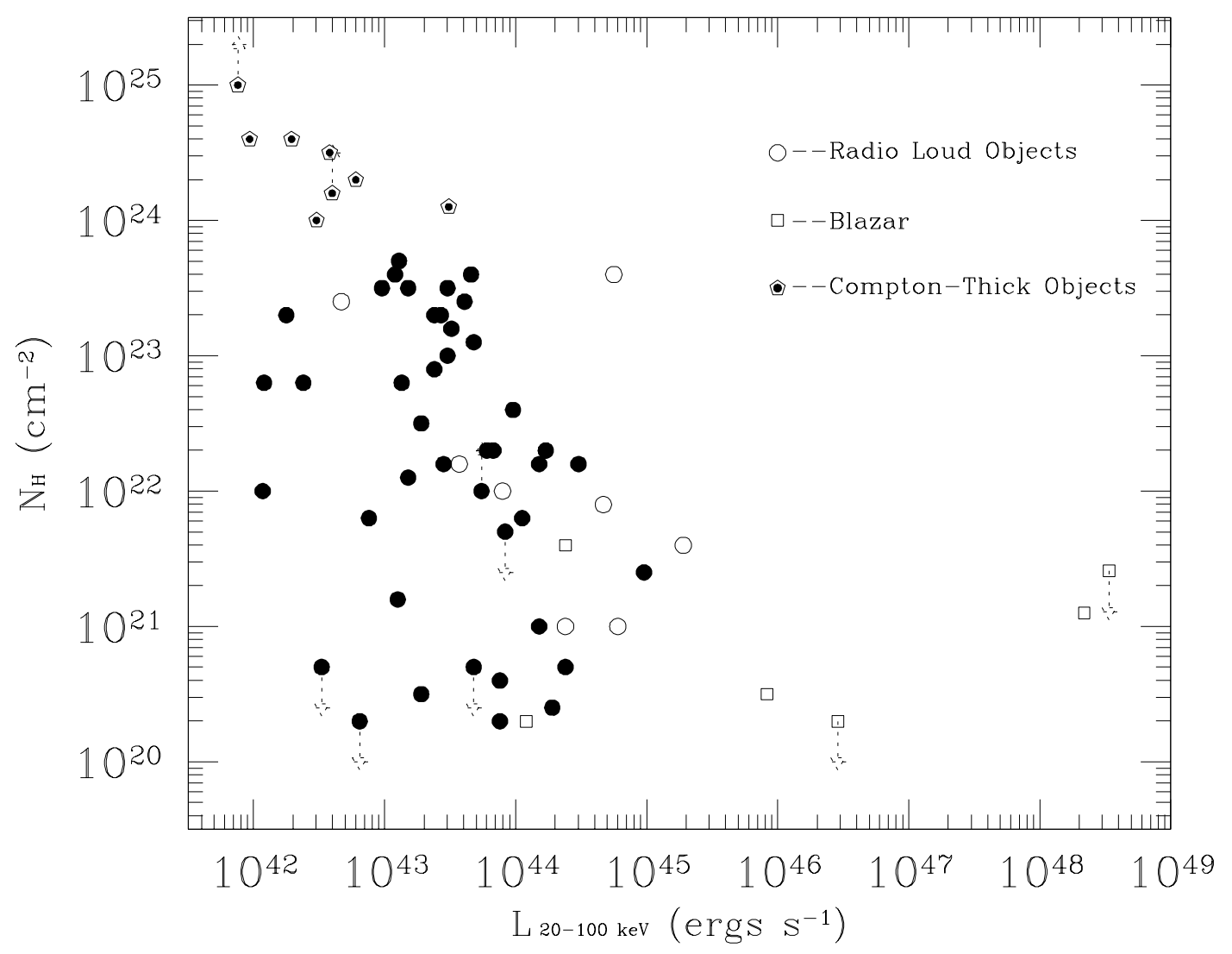

Fig. 4.- The X-ray absorption column density $N_{H}$ versus the INTEGRAL luminosity for the 65 AGNs in the combined sample. 


\section{Table 1. Soft Gamma-ray selected AGNs with an updated list of $N_{H}$}

\begin{tabular}{lccccc}
\hline \hline \multicolumn{1}{c}{ Source } & $\mathrm{z}$ & Type $^{c}$ & $\mathrm{~N}_{H}$ & Ref. & $\log L$ \\
\hline Bassani et al. 06 & INTEGRAL & & & & $\log \mathrm{L}_{20-100 \mathrm{eV}}$ \\
\hline QSO B0241+62 & 0.044 & RQ & 22.2 & 1 & 44.48 \\
$\ldots$. & & & & & \\
\hline Markwardt et al. 2005 & SWIFT & & & & $\log \mathrm{L}_{14-195 \mathrm{keV}^{-0.018}}$ \\
\hline Mrk 348 & 0.0150 & RQ & 23.1 & 14 & 43.68
\end{tabular}

Note. - The complete version of this table is in the electronic edition of the Journal. The printed edition contains only a sample. ${ }^{a}$ Sources included by both Bassani et al. 2006 and Markwardt et al. 2005. ${ }^{b}$ QSO 0836+710. ${ }^{c}$ RQ $=$ radio quiet; $\mathrm{RL}=$ radio loud; $\mathrm{Bl}=$ blazar; Co = Compton thick. References.-(1) B06; (2) Matt et al. 2006; (3) Dewangan, Griffiths \& Schurch 2003; (4) Beckmann et al. 2005; (5) Beckmann et al. 2004; (6) M05; (7) Matt et al. 2004; (8) Steenbrugge et al. 2003; (9) Steenbrugge et al. 2005; (10) Matsumoto et al. 2004; (11) Lewis et al. 2005; (12) Bianchi et al. 2005; (13) Foschini

et al. 2006; (14) Akylas et al. 2002; (15) Risaliti et al. 2005; (16) Ballantyne et al. 2004; (17) Vaughan et al. 2004; (18)

Colber et al. 2005; (19) Jiang, Wang \& Wang 2006; (20) Yaqoob et al. 2005; (21) Costantini et al. 2005; (22) Gibson et al. 2005; (23) Turner et al. 2000; (24) Bianchi et al. 2003 